\title{
High-pressure balloon dilation for male anterior urethral stricture: single-center experience*
}

\author{
Shi-cheng YU, Hai-yang WU, Wei WANG, Li-wei XU, Guo-qing DING, Zhi-gen ZHANG, Gong-hui LI ${ }^{\dagger *}$ \\ (Department of Urology, Sir Run Run Shaw Hospital, School of Medicine, Zhejiang University, Hangzhou 310016, China) \\ †E-mail: ligonghui1970@hotmail.com \\ Received Mar. 1, 2016; Revision accepted May 4, 2016; Crosschecked Aug. 8, 2016
}

\begin{abstract}
Objectives: We retrospectively reviewed the urethral stricture cases treated in our tertiary center, and assessed the safety and feasibility of the high-pressure balloon dilation (HPBD) technique for anterior urethral stricture. Methods: From January 2009 to December 2012, a total of 31 patients with anterior urethral strictures underwent HPBD at our center, while another 25 cases were treated by direct vision internal urethrotomy (DVIU). Patient demographics, stricture characteristics, surgical techniques, and operative outcomes were assessed and compared between the two groups. The Kaplan-Meier survival analysis was applied to evaluate the stricture-free rate for the two surgical techniques. Results: The operation time was much shorter for the HPBD procedure than for the DVIU $((13.19 \pm 2.68)$ min vs. $(18.44 \pm 3.29) \mathrm{min}, P<0.01)$. For the HPBD group, the major postoperative complications as urethral bleeding and urinary tract infection (UTI) were less frequently encountered than those in DVIU (urethral bleeding: $2 / 31$ vs. $8 / 25, P=0.017$; UTI: $1 / 31$ vs. $6 / 25 P=0.037$ ). The Kaplan-Meier survival analysis showed that there was no significant difference in stricture-free rate at 36 months between the two groups $(P=0.21$, hazard ratio $(\mathrm{HR})=0.65,95 \%$ confidence interval $(\mathrm{Cl}): 0.34$ to 1.26$)$. However, there was a significantly higher stricture-free survival in the HPBD group at 12 months $(P=0.02, \mathrm{HR}=0.35,95 \% \mathrm{Cl}: 0.14$ to 0.87$)$, which indicated that the stricture recurrence could be delayed by using the HPBD technique. Conclusions: HPBD was effective and safe and it could be considered as an alternative treatment modality for anterior urethral stricture disease.
\end{abstract}

Key words: Urethral stricture, High-pressure balloon dilation, Urethrotomy http://dx.doi.org/10.1631/jzus.B1600096

CLC number: R69

\section{Introduction}

Male urethral stricture disease is one of the most difficult maladies known to urologists with an estimated incidence ranged from $0.1 \%$ to $0.6 \%$ (Santucci et al., 2007; Mundy and Andrich, 2011). Historically, urethral strictures were associated with chronic or recurrent urethral infection such as gonorrhea, but nowadays the major causes of strictures in most

\footnotetext{
Corresponding author

* Project supported by the Medical Health Program of Zhejiang Province (No. 2014ZDA011) and the Zhejiang Provincial Natural Science Foundation of China (No. 2015KYB222)

(D) ORCID: Shi-cheng YU, http://orcid.org/0000-0003-4617-3065

(C) Zhejiang University and Springer-Verlag Berlin Heidelberg 2016
}

communities are post-traumatic, iatrogenic, and idiopathic (Mundy and Andrich, 2011).

The surgical treatment of urethral stricture remains controversial. In general, reconstructive surgery either with end-to-end anastomosis or substitution urethroplasty is considered the most successful option, but it requires surgical expertise and is associated with a high complication rate (Gupta et al., 2009). Endoscopic surgery like direct vision internal urethrotomy (DVIU) is currently the most commonly performed treatment for newly diagnosed urethral stricture. The popularity of this procedure has been attributed to the ease of performance and a perceived low complication rate (Bullock and Brandes, 2007; van Leeuwen et al., 2011). Despite its widespread 
acceptance as the first-line option, the failure rate after initial DVIU is reported to be $50 \%-89 \%$ (Tonkin and Jordan, 2009; Santucci and Eisenberg, 2010; Anger et al., 2011a; 2011b). By incising the urethra via urethrotomy, the vascularity within the underlying corpus spongiosum might be destroyed during the procedure, which could exacerbate the ischemic spongiofibrosis and lead to the recurrence of stricture. In light of this, the place of DVIU in the treatment algorithm for male urethral stricture disease has been questioned (Heyns et al., 1998; Hudak et al., 2012).

High-pressure balloon dilation (HPBD) is an effective and safe procedure that has been well documented in the management of ureteral stricture (Lewis-Russell et al., 2004; Kuntz et al., 2015). We have applied HPBD in a series of patients with anterior urethral strictures. In our experience, it is a good option as the initial treatment for anterior urethral stricture. Here we retrospectively review the urethral stricture cases treated by the HPBD technique at our center, and assess the safety and feasibility of this technique as an alternative to traditional endoscopic surgeries.

\section{Materials and methods}

\subsection{Clinical data and research methods}

After receiving approval by the Institutional Review Board of Sir Run Run Shaw Hospital (China), a retrospective chart review was conducted for patients of urethral strictures who had underwent endoscopic surgery at our tertiary center between January 2009 and December 2012. Patients with a history of hypospadias repair, lichen sclerosis, prior urethroplasty, or recurrent urethral stricture disease were excluded from the analysis, as well as men with posterior urethral strictures. Thus, we had identified a highly select homogenous subset of patients with anterior urethral strictures who were most likely to be considered as ideal candidates for endoscopic surgery. Patients were grouped by the surgical technique used in the procedure into the HPBD group and the DVIU group. Patient demographics, stricture characteristics, surgical technique, and operative outcomes were assessed and compared between the two groups.

Patients were initially assessed with uroflowmetry, cystoscopy, retrograde urethrogram, and/or voiding cystourethrogram. Patients' characteristics and causes of stricture were obtained by reviewing the electronic medical record. The diagnosis of urethral stricture was established via routine cystoscopy and retrograde urethrogram. Stricture length and location were determined from the preoperative urethrogram or operative report based on intraoperative calibration. All the procedures of HPBD and DVIU had been performed by two senior surgeons at our center. All patients were provided with information on the surgical procedure prior to surgery, and an informed consent was obtained from each patient.

The major complications associated with treatment, such as urethral bleeding and urinary tract infection (UTI), were determined by reviewing the electronic medical record. Uroflowmetry, retrograde urethrogram, and/or voiding cystourethrogram were performed at postoperative catheter removal. After surgery, patients were followed up at regular 3-month intervals for 3 years, and uroflowmetry was performed at each follow-up visit. Additional office visits were made if the patients had relapsing symptoms of stricture. Retrograde urethrogram and cystoscopy were performed as indicated based on patient's recurrent voiding symptoms, a decrease of urinary flow rate, or an obstructive voiding curve on uroflowmetry.

Primary success was defined as freedom from additional treatments except for short-term intermittent dilation. Failure of the treatment was defined as stricture recurrence that could not be resolved by adjuvant instrumentation including intermittent dilation, and patients finally referred to urethroplasty. In our study, the objective sign of recurrence was confirmed by definitive radiographic or cystoscopic evidence of stricture.

\subsection{Surgical technique}

The procedure of HPBD was performed under spinal anesthesia, and the patient was placed in the lithotomy position. The first step was to obtain imaging studies of the stricture segment. Routine intraoperative retrograde urethrogram will help guide the procedure. An 8/9.5 F ureteroscope was inserted carefully and advanced gently with meticulous inspection of the urethra up to the point of the stricture (Fig. 1a). A 0.035-inch hydrophilic guide wire was introduced through the stricture segment down to the 
bladder neck. Then a standard pre-inflated 7 F, $60 \mathrm{~mm}$ balloon catheter (X-Force ${ }^{\mathrm{TM}}, \mathrm{C} . \mathrm{R}$. Bard Inc., USA) was inserted over the guide wire under direct vision guidance (Fig. 1b). Using a 10-ml syringe (EAGLETM C.R. Bard Inc., USA), the balloon was inflated with radiological contrast. The urethral stricture was dilated under fluoroscopic guidance for $5 \mathrm{~min}$ until the balloon notch image disappeared when the pressure was increased up to $16-20 \mathrm{~atm}(1 \mathrm{~atm}=101325 \mathrm{~Pa})$ with the maximum diameter of $21 \mathrm{~F}$. The DVIU procedure was performed using a modified technique including multiple radial incisions at the 3,6,9, and 12 o'clock positions with a cold knife. A 20 F Foley catheter was kept indwelling in all patients, which was removed 2-3 weeks later after surgery.
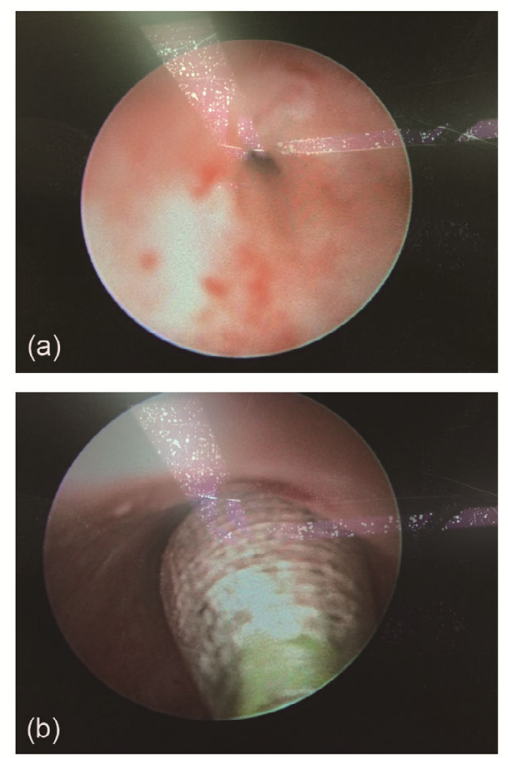

Fig. 1 Appearance of urethral stricture at the beginning of the HPBD procedure (a) and high-pressure balloon catheter inserted through the stricture segment over the guide wire (b)

\subsection{Statistical analysis}

Data were tabulated and analyzed using the SPSS $^{\circledR}$ package 19.0 (SPSS, Inc., USA) and GraphPad Prism ${ }^{\circledR} 5.01$ (GraphPad Software, Inc., USA) software. Univariate analyses of continuous and categorical variables were done using the independent sample $t$-test and Fisher exact test together with Chi-square test, respectively, with statistical significance considered at $P<0.05$. The Kaplan-Meier survival analysis was applied to evaluate the stricturefree rate for the two groups, and the log-rank test was performed to compare the efficacy of different surgical techniques. Patients without stricture recurrence were considered as survivors, and the survival time was regarded as the time from initial treatment until the stricture recurrence had been confirmed.

\section{Results}

Of the 109 urethral stricture cases treated during the study period, 62 were anterior urethral strictures that met the study inclusion criteria. A total of 35 patients underwent HPBD, with 4 cases lost to follow-up, leaving a remainder of 31 patients to be analyzed. And the other 27 cases were treated by DVIU with 2 patients excluded from study due to loss during follow-up.

Patient's demographics and clinical features of urethral strictures were summarized (Table 1). The median age of the HPBD group was 49 (range 3267 ) years while that of the DVIU group was 44 (range 24-71) years. The most common causes of urethral stricture were posttraumatic and iatrogenic in both groups (HPBD vs. DVIU: posttraumatic 21/31 vs. $13 / 25$, iatrogenic $7 / 31$ vs. $9 / 25$ ). The majority of stricture lesions in both groups were located in the bulbar urethra (HPBD vs. DVIU: 24/31 vs. 21/25), and most of the cases were shorter than $1 \mathrm{~cm}$ in length (HPBD vs. DVIU: $26 / 31$ vs. 22/25), making the patients ideal candidates for endoscopic surgery.

Table 1 Patient's demographics

\begin{tabular}{lccc}
\hline \multicolumn{1}{c}{ Characteristics } & $\begin{array}{c}\text { HPBD } \\
(n=31)\end{array}$ & $\begin{array}{c}\text { DVIU } \\
(n=25)\end{array}$ & $P$-value $^{*}$ \\
\hline Age (median, range) & $49,32-67$ & $44,24-71$ & 0.065 \\
Etiology & & & \\
$\quad$ Posttraumatic & $21(67.7 \%)$ & $13(52.0 \%)$ & 0.536 \\
$\quad$ Iatrogenic & $7(22.6 \%)$ & $9(36.0 \%)$ & \\
$\quad$ Idiopathic & $1(3.2 \%)$ & $2(8.0 \%)$ & \\
$\quad$ Infection & $2(6.5 \%)$ & $1(4.0 \%)$ & \\
Location & & & \\
$\quad$ Penile & $7(22.6 \%)$ & $4(16.0 \%)$ & 0.737 \\
$\quad$ Bulbar & $24(77.4 \%)$ & $21(84.0 \%)$ & \\
Length & & & \\
$\leq 1 \mathrm{~cm}$ & $26(83.9 \%)$ & $22(88.0 \%)$ & 0.720 \\
$>1 \mathrm{~cm}$ & $5(16.1 \%)$ & $3(12.0 \%)$ & \\
\hline
\end{tabular}

Data are expressed as number (percentage), except age. ${ }^{*}$ Student's $t$-test for continuous variables; Fisher exact test and Chi-square test for categorical variables 
The operation time was much shorter for HPBD than for DVIU (HPBD vs. DVIU: $(13.19 \pm 2.68) \mathrm{min}$ vs. $(18.44 \pm 3.29) \mathrm{min}, P<0.01)$, which indicated that the HPBD procedure was much easier to perform. The major postoperative complications were urethral bleeding and UTI. For the HPBD group, only 2 patients $(6.45 \%)$ reported one episode of urethral bleeding and the other $29(93.55 \%)$ never experienced any. However, in the DVIU group, 8 patients $(32.00 \%)$ had urethral bleeding while 17 patients $(68.00 \%)$ never encountered it $(P=0.017)$. One patient $(3.23 \%)$ in the HPBD group experienced UTI, while 30 patients $(96.77 \%)$ never encountered any infection. In contrast, 6 patients $(24.00 \%)$ had UTI and the other 19 ones $(76.00 \%)$ did not encountered any in the DVIU group $(P=0.037)$ as shown in Table 2 .

Table 2 Surgical technique and complications

\begin{tabular}{cccc}
\hline \multirow{2}{*}{ Group } & \multirow{2}{*}{$\begin{array}{c}\text { Operation time } \\
(\mathrm{min})\end{array}$} & \multicolumn{2}{c}{ Complication } \\
\cline { 3 - 4 } & & Bleeding & UTI \\
\hline HPBD $(n=31)$ & $13.19 \pm 2.68$ & $2(6.45 \%)$ & $1(3.23 \%)$ \\
DVIU $(n=25)$ & $18.44 \pm 3.29$ & $8(32.00 \%)$ & $6(24.00 \%)$ \\
\hline$P^{*}$ & $<0.01$ & 0.017 & 0.037 \\
\hline
\end{tabular}

Data are expressed as mean \pm standard deviation (SD) or number (percentage). ${ }^{*}$ Student $t$-test for continuous variables. Fisher exact test and Chi-square test for categorical variables

The initial surgical outcomes were assessed at postoperative catheter removal. Uroflowmetry showed that the maximum urinary flow rate $\left(Q_{\max }\right)$ after initial treatment was $(17.85 \pm 3.86)$ and $(16.29 \pm$ 2.95) $\mathrm{ml} / \mathrm{s}$ for HPBD and DVIU, respectively, whereas the $Q_{\max }$ before surgery was $(4.94 \pm 1.77)$ and $(5.88 \pm 1.81) \mathrm{ml} / \mathrm{s}$, respectively. Patients had been followed up periodically after surgery for 5-36 months with a median of 14.75 months. The overall result showed that 20 patients $(64.50 \%)$ of the HPBD group failed in treatment and finally referred to urethroplasty due to stricture recurrence, while the number in the DVIU group was $18(72.00 \%)$ as shown in Table 3.

Table 3 Operative outcomes

\begin{tabular}{cccc}
\hline \multirow{2}{*}{ Group } & \multicolumn{2}{c}{$Q_{\max }(\mathrm{ml} / \mathrm{s})$} & \\
\cline { 2 - 3 } & $\begin{array}{c}\text { Before } \\
\text { operation }\end{array}$ & $\begin{array}{c}\text { After } \\
\text { operation }\end{array}$ & \\
\hline HPBD $(n=31)$ & $4.94 \pm 1.77$ & $17.85 \pm 3.86$ & $20(64.50 \%)$ \\
DVIU $(n=25)$ & $5.88 \pm 1.81$ & $16.29 \pm 2.95$ & $18(72.00 \%)$ \\
\hline
\end{tabular}

$Q_{\text {max }}$ : maximum urinary flow rate. Data are expressed as mean \pm standard deviation (SD) or number (percentage)
The Kaplan-Meier survival analysis showed that the estimated stricture-free rate at 36 months was $35.48 \%$ after HPBD and $28.00 \%$ after DVIU. The median time to stricture recurrence was 17 months after HPBD and 11 months after DVIU. However, the log-rank test demonstrated that there was no significant difference in stricture-free rate between the two groups $(P=0.21$, hazard ratio $(\mathrm{HR})=0.65,95 \%$ confidence interval (CI) for HR: 0.34-1.26; Fig. 2a). For the purpose of further analysis, we evaluated the stricture-free survival status at 12 months of the two groups. The estimated stricture-free rate at 12 months was $77.42 \%$ after HPBD and $48.00 \%$ after DVIU, which showed a significantly higher stricture-free survival in the HPBD group $(P=0.02, \mathrm{HR}=0.35,95 \%$ CI for HR: 0.14-0.87; Fig. 2b). The survival analysis revealed that, although there was no statistically significant difference in the overall stricture-free survival, stricture recurrence could be delayed by using the HPBD technique.
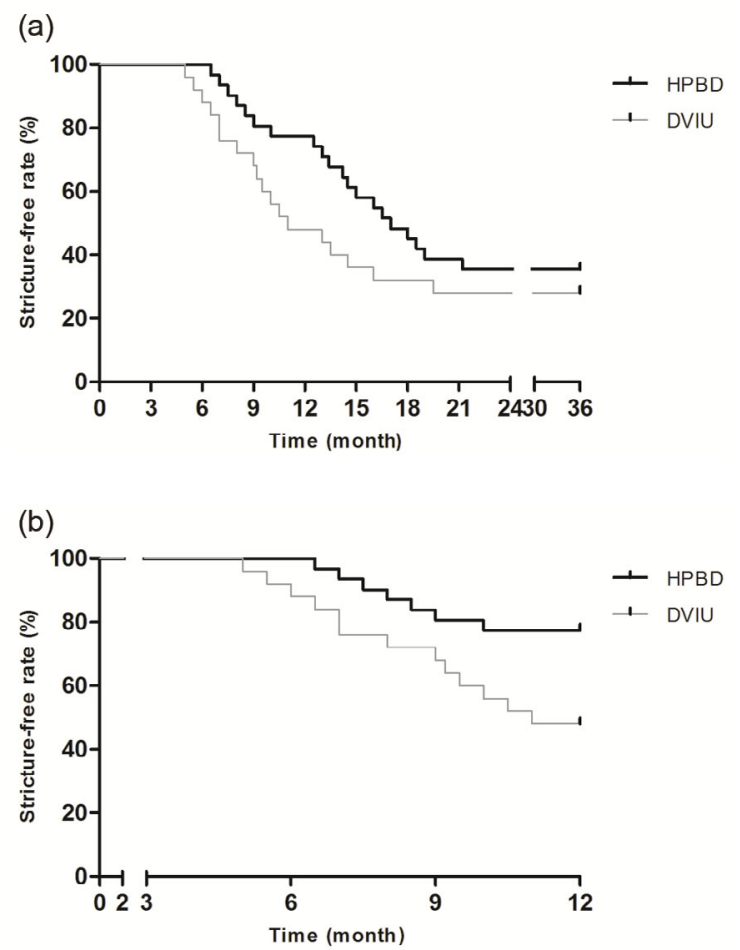

Fig. 2 Kaplan-Meier curves predicting stricture-free rate after HPBD and DVIU

(a) Survival analysis showing no significant difference at 36 months $(P=0.21)$. (b) Survival analysis showing significantly higher stricture-free rate in the HPBD group at 12 months $(P=0.02)$ 


\section{Discussion}

Despite the alarmingly high stricture recurrence rate of nearly $50 \%$, DVIU remains the most commonly applied procedure due to its relative simplicity and ease of performance (Ishigooka et al., 1995; Santucci and Eisenberg, 2010). On the contrary, urethroplasty was traditionally considered a difficult surgical procedure and reserved for much more complex cases, although advanced with better longterm cure rates (Singh et al., 2011). However, it has been demonstrated that endoscopic surgery was superior to urethroplasty as long as the stricture did not exceed $2 \mathrm{~cm}$ in length, given its low morbidity and rapid convalescence (Wright et al., 2006; Hameed et al., 2011; Veeratterapillay and Pickard, 2012; Tinaut-Ranera et al., 2014).

The principle of conventional DVIU is to achieve epithelial regrowth by the incision of the scar tissue. The major disadvantage of DVIU is that the depth of the scar tissue cannot be estimated accurately during the procedure, resulting in imprecise incision of the scar tissue. It is possible that the incision of the urethral stricture may not reach the healthy tissue, which cannot minimize the stricture recurrence effectively. On the other hand, by incising the urethra via a cold knife, the underlying corpus spongiosum might be injured, which would lead to postoperative hemorrhage. The destroyed vascularity within the corpus spongiosum and focal urinary extravasation through fissures on the mucosa might exacerbate the spongiofibrosis and finally turn to stricture recurrence (Isen and Nalcacioglu, 2015).

HPBD has been well documented in the management of ureteral stricture (Lewis-Russell et al., 2004; Kuntz et al., 2015). The HPBD procedure achieves dilation via radial cracking and expansion of the surrounding scar tissue. The high-pressure balloon can exert over 16-20 atm of pressure, which can adequately address the incomplete dilation. During the HPBD procedure, radial expansion of a balloon dilator is achieved by distribution of the radial force along the balloon span, and it has been proposed that these radial forces are directed perpendicular to the mucosa, which results in less shearing forces on the mucosal surface and is associated with reduced tissue trauma (Yu et al., 2011; Parente et al., 2013). Thus, the vascularity within the underlying corpus spongiosum could be well preserved, thereby resulting in less frequent postoperative hemorrhage and spongiofibrosis.

In our study, the HPBD procedure was much easier to perform (operation time: $(13.19 \pm 2.68) \mathrm{min}$ vs. (18.44 \pm 3.29$) \mathrm{min}$, HPBD vs. DVIU, respectively; $P<0.01)$ with fewer postoperative complications (urethral bleeding and UTI: $2 / 31$ vs. $8 / 25$ and 1/31 vs. $6 / 25$, HPBD vs. DVIU, respectively). Although the overall stricture-free survival showed that the longterm cure rates were equal in the two groups, most of the recurrent diseases in the HPBD group had occurred 12 months later after the initial treatment. It is estimated that stricture recurrence could be delayed by using the HPBD technique.

The limitations of our work include the small number of patients and the retrospective nature of the study. The results should be confirmed in a prospective study and a large number of patients, which will allow us to perform an analysis through subclassifications of patients by comorbidities or cause of strictures.

\section{Conclusions}

HPBD might be an alternative for the treatment modality of anterior urethral stricture disease. Given its technical ease, low acute morbidity, and rapid convalescence, HPBD could be considered a temporizing measure, especially for candidates with shorter length stricture disease or until further reconstructive surgery can be planned.

\section{Compliance with ethics guidelines}

Shi-cheng YU, Hai-yang WU, Wei WANG, Li-wei XU, Guo-qing DING, Zhi-gen ZHANG, and Gong-hui LI declare that they have no conflict of interest.

All procedures followed were in accordance with the ethical standards of the responsible committee on human experimentation (institutional and national) and with the Helsinki Declaration of 1975, as revised in 2008 (5). Informed consent was obtained from all patients for being included in the study.

\section{References}

Anger, J.T., Buckley, J.C., Santucci, R.A., et al., 2011a. Trends in stricture management among male medicare beneficiaries: underuse of urethroplasty? Urology, 77(2): $481-485$ http://dx.doi.org/10.1016/j.urology.2010.05.055 
Anger, J.T., Scott, V.C., Sevilla, C., et al., 2011b. Patterns of management of urethral stricture disease in the veterans affairs system. Urology, 78(2):454-458. http://dx.doi.org/10.1016/j.urology.2010.12.081

Bullock, T.L., Brandes, S.B., 2007. Adult anterior urethral strictures: a national practice patterns survey of board certified urologists in the United States. J. Urol., 177(2): 685-690. http://dx.doi.org/10.1016/j.juro.2006.09.052

Gupta, N.P., Mishra, S., Dogra, P.N., et al., 2009. Outcome of end-to-end urethroplasty: single-center experience. Urol. Int., 82(2):179-182. http://dx.doi.org/10.1159/000200796

Hameed, A., Mohammed, A., Nasir, S., et al., 2011. Management of bulbar urethral strictures: review of current practice. Can. J. Urol., 18(3):5676-5682.

Heyns, C.F., Steenkamp, J.W., de Kock, M.L., et al., 1998. Treatment of male urethral strictures: is repeated dilation or internal urethrotomy useful? J. Urol., 160(2):356-358. http://dx.doi.org/10.1016/S0022-5347(01)62894-5

Hudak, S.J., Atkinson, T.H., Morey, A.F., 2012. Repeat transurethral manipulation of bulbar urethral strictures is associated with increased stricture complexity and prolonged disease duration. J. Urol., 187(5):1691-1695. http://dx.doi.org/10.1016/j.juro.2011.12.074

Isen, K., Nalcacioglu, V., 2015. Direct vision internal urethrotomy by using endoscopic scissors. Int. Urol. Nephrol., 47(6):905-908. http://dx.doi.org/10.1007/s11255-015-0960-x

Ishigooka, M., Tomaru, M., Hashimoto, T., et al., 1995. Recurrence of urethral stricture after single internal urethrotomy. Int. Urol. Nephrol., 27(1):101-106. http://dx.doi.org/10.1007/BF02575227

Kuntz, N.J., Neisius, A., Tsivian, M., et al., 2015. Balloon dilation of the ureter: a contemporary review of outcomes and complications. J. Urol., 194(2):413-417. http://dx.doi.org/10.1016/j.juro.2015.02.2917

Lewis-Russell, J.M., Natale, S., Hammonds, J.C., et al., 2004. Ten years' experience of retrograde balloon dilatation of pelviureteric junction obstruction. BJU Int., 93(3):360-363. http://dx.doi.org/10.1111/j.1464-410X.2003.04617.x

Mundy, A.R., Andrich, D.E., 2011. Urethral strictures. BJU Int. 107(1):6-26. http://dx.doi.org/10.1111/j.1464-410X.2010.09800.x

Parente, A., Angulo, J.M., Romero, R.M., et al., 2013. Management of ureteropelvic junction obstruction with high-pressure balloon dilatation: long-term outcome in 50 children under 18 months of age. Urology, 82(5): 1138-1143. http://dx.doi.org/10.1016/j.urology.2013.04.072

Santucci, R., Eisenberg, L., 2010. Urethrotomy has a much lower success rate than previously reported. J. Urol., 183(5):1859-1862. http://dx.doi.org/10.1016/j.juro.2010.01.020

Santucci, R.A., Joyce, G.F., Wise, M., 2007. Male urethral stricture disease. J. Urol., 177(5):1667-1674. http://dx.doi.org/10.1016/j.juro.2007.01.041

Singh, O., Gupta, S.S., Arvind, N.K., 2011. Anterior urethral strictures: a brief review of the current surgical treatment.
Urol. Int., 86(1):1-10.

http://dx.doi.org/10.1159/000319501

Tinaut-Ranera, J., Arrabal-Polo, M.A., Merino-Salas, S., et al., 2014. Outcome of urethral strictures treated by endoscopic urethrotomy and urethroplasty. Can. Urol. Assoc. J., 8(1-2):E16-E19. http://dx.doi.org/10.5489/cuaj.1407

Tonkin, J.B., Jordan, G.H., 2009. Management of distal anterior urethral strictures. Nat. Rev. Urol., 6(10):533-538. http://dx.doi.org/10.1038/nrurol.2009.181

van Leeuwen, M.A., Brandenburg, J.J., Kok, E.T., et al., 2011. Management of adult anterior urethral stricture disease: nationwide survey among urologists in the Netherlands. Eur. Urol., 60(1):159-166. http://dx.doi.org/10.1016/j.eururo.2011.03.016

Veeratterapillay, R., Pickard, R.S., 2012. Long-term effect of urethral dilatation and internal urethrotomy for urethral strictures. Curr. Opin. Urol., 22(6):467-473. http://dx.doi.org/10.1097/MOU.0b013e32835621a2

Wright, J.L., Wessells, H., Nathens, A.B., et al., 2006. What is the most cost-effective treatment for 1 to $2-\mathrm{cm}$ bulbar urethral strictures: societal approach using decision analysis. Urology, 67(5):889-893. http://dx.doi.org/10.1016/j.urology.2005.11.003

Yu, H.L., Ye, L.Y., Lin, M.H., et al., 2011. Treatment of benign ureteral stricture by double $\mathrm{J}$ stents using highpressure balloon angioplasty. Chin. Med. J. (Engl.), 124(6):943-946.

\section{中文概要}

题 目: 高压球囊扩张治疗男性前尿道狭窄: 单中心的临 床经验

目 的: 评估高压球囊扩张治疗男性前尿道狭窄的有效性 和安全性。

创新点: 既往高压球囊扩张技术已成熟应用于输尿管狭窄 的临床治疗, 我们将这一技术拓宽应用于男性前 尿道狭窄的治疗, 并证明高压球囊扩张治疗前尿 道狭窄同样安全、有效。

方 法: 采用回顾性病例对照研究, 收集并分析了 31 例 接受高压球囊扩张和 25 例接受直视下尿道内切 开术治疗的前尿道患者的病例资料, 比较两组患 者的手术时间、手术难易程度、术后并发症（出 血和感染)、术后 1 年狭窄复发率和术后 3 年狭 窄复发率等相关指标。

结 论: 高压球囊扩张组的手术时间和术后并发症发生率 要优于直视下尿道内切开术组, 两组患者的远期 尿道狭窄复发率无统计学差异, 但高压球囊扩张 组的术后 1 年狭窄复发率明显低于直视下尿道 内切开术组。高压球囊扩张治疗男性前尿道狭窄 是安全、有效的, 且近期疗效要优于传统腔内治 疗方式。

关键词: 尿道狭窄; 高压球囊扩张; 尿道内切开术 